Martin Hellwig, Gerhard Schick

\section{Krise und Reformbedarf der BaFin}

Der Skandal um Wirecard hat gezeigt, dass es um die deutsche Finanzaufsicht schlecht bestellt ist. Die nun begonnenen Reformen gehen in die richtige Richtung. Wesentliche Probleme werden aber nach wie vor nicht angegangen. Man muss daher mit weiteren Krisen und Skandalen rechnen.

Die Qualität der Finanzaufsicht war schon einmal ein Thema, in der globalen Finanzkrise. Diese traf Deutschland besonders stark - und das, obwohl Deutschland nicht zu den Epizentren der Krise gehörte. Die Kosten für den deutschen Steuerzahler lagen in der Größenordnung von 80 Mrd. Euro (Hellwig, 2017). Die Bundesregierung vertrat damals die Auffassung, die Krise sei aus den USA gekommen, zuerst mit der Herstellung „toxischer“ Wertpapiere, dann mit der Lehman-Insolvenz. Die deutschen Finanzinstitute seien nur Opfer gewesen, so der Bundesfinanzminister vor dem Bundestagsuntersuchungsausschuss zu Hypo Real Estate.

Anders als in den USA, Großbritannien, Island oder der Schweiz hat es keine Untersuchung der deutschen Betroffenheit durch die Finanzkrise gegeben. Die Bundesregierung hat derartige Analysen sogar aktiv behindert, indem sie die Herausgabe von Informationen verweigerte, auch auf offizielle Anfragen der Fraktion Bündnis 90/ Die Grünen im Bundestag. Erst 2017 sorgte ein Urteil des Bundesverfassungsgerichts für Abhilfe.

Im Folgenden skizzieren wir zunächst das Versagen der Aufsicht im Vorfeld der Finanzkrise und seither. Sodann diskutieren wir, warum die Reaktion der Bundesregierung auf den Wirecard-Skandal nicht ausreicht, und formulieren Desiderata für eine grundlegende Reform.

\section{Aufsichtsversagen im Vorfeld der Finanzkrise}

Die Schieflagen deutscher Banken in der Finanzkrise gingen auf vier massive Fehlentwicklungen zurück:

1. Der Eigenkapitaleinsatz war extrem niedrig, bei den großen Banken von 1,5\% bis $4 \%$ der Anlagen.

2. Banken benutzten Zweckgesellschaften, Briefkopffirmen ohne Eigenkapital, um Finanzanlagen zu tätigen, ohne sie in der eigenen Bilanz zu führen.

(c) Der/die Autor:in(nen) 2021. Open Access: Dieser Artikel wird unter der Creative Commons Namensnennung 4.0 International Lizenz veröffentlicht (creativecommons.org/licenses/by/4.0/deed.de).

Open Access wird durch die ZBW - Leibniz-Informationszentrum Wirtschaft gefördert.
3. Große Banken finanzierten sich in großem Umfang kurzfristig über den Geldmarkt und kamen in Schwierigkeiten als dieser zusammenbrach.

4. Viele Anlagen taugten nicht viel, so etwa Hypotheken und Hypothekenverbriefungen in den USA, Irland und Spanien, Interbankenkredite in Irland und Griechenland, Schiffskredite weltweit.

Die 2017/18 gegebenen Antworten der Bundesregierung auf die Anfragen der Bundestagsfraktion Bündnis 90/Die Grünen von 2010 vermitteln folgende Eindrücke:

- Die Aufsicht war überwiegend untätig. Die Aufsichtsbehörden nahmen an Gremiensitzungen der Institute teil, haben aber nur sehr selten das Wort ergriffen. Anlassbezogene Aufsichtsgespräche und Sonderprüfungen gab es erst in der Krise.

- Die Aufsicht hat die Risikoeinschätzungen der Institute nicht hinterfragt, weder bei den Zweckgesellschaften noch bei der Konzentration von Risiken aufgrund hoher Korrelationen bei Immobilien- und Schiffskrediten noch bei Akquisitionen wie Depfa (HRE) und Hypo Group Alpe Adria (BayernLB). In all diesen Fällen waren die Risiken erkennbar exorbitant.

- Die Aufsicht hatte kein Auge für Systemzusammenhänge und für Korrelationen von Einzelrisiken einerseits und für die mit der Fristentransformation verbundenen Risiken andererseits.

Die Aufsicht scheint vor allem ein kooperatives Verhältnis zu den Banken angestrebt zu haben; so hieß es in der Verhandlung beim Bundesverfassungsgericht, eine Veröffentlichung der geforderten Information könnte das Vertrauensverhältnis zu den Banken zerstören.

Prof. Dr. Martin Hellwig ist emeritierter Direktor am Max-Planck-Institut zur Erforschung von Gemeinschaftsgütern in Bonn.

Dr. Gerhard Schick ist Vorstand des Vereins Bürgerbewegung Finanzwende. 
Das Verhalten der Aufsicht war auch stark legalistisch geprägt. Man tat so, als seien die Zweckgesellschaften eigenständige Unternehmen. Dass die Muttergesellschaften mit Liquiditätsgarantien für diese Zweckgesellschaften selbst im Risiko standen, wurde „übersehen“.

Die Rechtsnormen enthalten Generalklauseln, die die Behörde ermächtigen, übermäßiges Eingehen von Risiken durch Banken zu verhindern. Daher hätte die BaFin die Banken zwingen können, die Zweckgesellschaften - oder wenigstens die Garantien - in ihre eigene Rechnungslegung aufzunehmen und entsprechende Risikovorsorge zu betreiben. Das hätte auch deshalb nahe gelegen, weil Basel II, die 2004 verabschiedete Reform des Basler Abkommens über Bankenaufsicht, den bisherigen Umgang mit Zweckgesellschaften untersagt hatte, sodass die laufende Praxis ohnehin beendet werden musste. In Frankreich beispielsweise sind die Behörden so vorgegangen (Thiemann, 2012).

Möglicherweise hat die Aufsicht mit ihrer legalistischen Vorgehensweise aber den Wünschen des vorgesetzten Ministeriums entsprochen. Ein hochrangiger Beamter des BMF hatte 2006 in einem Vortrag die Entwicklung von Verbriefungen und die Verwendung von Zweckgesellschaften zum Halten von Verbriefungen ausdrücklich begrüßt und angekündigt, das BMF werde das Seinige tun, um dazu beizutragen, dass deutsche Institute sich prominent an diesen Entwicklungen beteiligten (Asmussen, 2006). Das könnte bedeuten, dass es eine Weisung des vorgesetzten Ministeriums gegeben hat, an die die Behörde sich halten musste. Aber auch ohne explizite Weisung des BMF ist denkbar, dass die Behörde im Wissen um diese Haltung lax gegenüber Zweckgesellschaften war und dies mit Legalismen begründete.

\section{CumEx, Wirecard und die Maßnahmen der Regierung}

Seit 2008 hat es keine Systemkrise mehr gegeben, dafür aber immer wieder Skandale, bei denen die BaFin wieder versagte. Zu nennen sind zahlreiche Betrugsfälle am so genannten grauen Kapitalmarkt, zuletzt P\&R, ferner der Cum-Ex-Skandal (Schick, 2020). Von diesem nahm die BaFin trotz der langen Dauer und des großen Volumens der Geschäfte keine Kenntnis; eine Aktivität der BaFin ließ sich erst wahrnehmen, als 2015/16 die Rückzahlungsforderungen des Staates die Maple-Bank insolvent werden ließen.

Und nun Wirecard! Wieder wies die BaFin jegliche Verantwortung von sich, Wirecard sei keine Bank, sondern ein Zahlungsdienstleister, nur eine Tochter in Bayern sei als Bank einzustufen und die sei vor Ort beaufsichtigt worden. Nach den Core Principles for Effective Banking Supervision des Basler Ausschusses für Bankenaufsicht hätte die Behörde allerdings den Gesamtkonzern und die Rolle der Bank-Tochter darin in den Blick nehmen müssen. ${ }^{1}$ Immerhin fühlte die BaFin sich betroffen genug, um ein Leerverkaufsverbot zu erlassen und mit Anzeigen gegen die Wirecard-Kritiker vorzugehen. Sie hätte stattdessen besser die Betrugsvorwürfe geprüft.

Die Bundesregierung reagierte zunächst wie üblich mit starker Rhetorik, Mini-Änderungen und einer Ablehnung der Übernahme von Verantwortung. Diesmal ging die Strategie allerdings nicht auf. Die Dimension des Skandals war zu groß, und aufgrund der Auseinandersetzung mit der Financial Times stand Deutschland auch international am Pranger.

Vor allem aber legte der Parlamentarische Untersuchungsausschuss die erschreckenden Fehlleistungen der BaFin, der Wirtschaftsprüferaufsicht APAS und der so genannten Bilanzpolizei DPR offen. Danach mussten die Chefs aller drei Behörden zurücktreten. Der Ausschuss zeigte schonungslos, dass die BaFin sich stärker als Verteidiger eines deutschen Finanzunternehmens gegen ausländische Angreifer sah, denn als Schützer heimischer Anleger und Steuerzahler gegen Betrüger.

Die Bundesregierung sah sich daher zu weiteren Reformen gezwungen: Das Finanzmarktintegritätsstärkungsgesetz (FISG) gibt der BaFin die primäre Zuständigkeit für Anlass- und Verdachtsprüfungen und unmittelbare Eingriffsbefugnisse für ausgelagerte Bankfunktionen. Ferner wurde den BaFin-Beschäftigten der Handel mit Finanzinstrumenten in weiten Teilen verboten. ${ }^{2}$ Ein Anfang 2021 verabschiedeter Sieben-Punkte-Plan sieht weiter vor: neue Spezialeinheiten für die Aufsicht über bestimmte komplexe Unternehmen, für Ad-hoc- und Sonderprüfungen vor Ort und für die Digitalisierung der BaFin, zusätzliches Personal für Bilanzprüfungen, ein Ausbau des Hinweisgebersystems und ein intensiverer Austausch mit Marktteilnehmer:innen, eine personelle und institutionelle Stärkung des Anleger- und Verbraucherschutzes und eine Zentralisierung der Leitungsstrukturen (BMF, 2021).

\section{Ein neues Paradigma für die Aufsicht}

Der Sieben-Punkte-Plan der Bundesregierung geht in die richtige Richtung. Jedoch beschränkt er sich auf kleine Schritte. Grundlegende Reformen werden wieder einmal aufgeschoben.

1 Principle 12 Essential Criteria (EC) 1 (wider group) in Verbindung mit Principle 8 EC 1 a.

2 Dass es eines solchen Verbots bedurfte, ist für sich schon skandalös. In anderen Ländern sind solche Verbote selbstverständlich, auch aufgrund des Straftatbestands des Insiderhandels. 


\section{Governance}

$\mathrm{Zu}$ reformieren sind vor allem die Zielsetzung und die Governance der Behörde. Das öffentliche Interesse, dem die BaFin verpflichtet sein sollte, ist nicht mit dem politischen Interesse der jeweiligen Regierung oder der Förderung der internationalen Wettbewerbsfähigkeit oder der sonstigen Interessen der Finanzinstitute gleichzusetzen.

Für Regulierung und Aufsicht im Finanzsektor gibt es zwei wesentliche Gründe. Zum einen gilt es, Systemrisiken zu vermeiden. Das Verhalten eines Instituts kann das Finanzsystem insgesamt gefährden, die Folgen des LehmanKonkurses sind dafür ein Beispiel. Zum anderen bedürfen Anleger des Schutzes. Der einzelne Anleger kann nicht angemessen und beizeiten prüfen und kontrollieren, was ein Finanzinstitut mit seinem Geld macht. Eine kompetente Aufsicht verhindert hier Missbräuche.

Beide Begründungen der Aufsichtstätigkeit beruhen auf der Überlegung, dass das von den Finanzinstituten freiwillig gewählte Verhalten Probleme verursacht. Ein gewisser Konflikt zwischen Behörde und Finanzinstituten liegt daher in der Natur der Sache. Das Kooperationsdenken der BaFin verdrängt diesen Tatbestand. Im Einzelnen sind folgende Aspekte der Governance zu ändern.

\section{Abhängigkeit vom Finanzministerium einschränken und transparent machen}

Die BaFin ist gegenüber dem Ministerium weisungsgebunden (Blank et al., 2021). Vertreter des Ministeriums beteuern regelmäßig, man mische sich nicht in Einzelfallentscheidungen ein, aber darüber gibt es keine Transparenz. ${ }^{3}$ In anderen Bereichen, z. B. der Wettbewerbspolitik und bei der Regulierung der Netzindustrien, unterscheiden die Rechtsnormen zwischen allgemeinen Weisungen und Einzelweisungen und sehen vor, dass allgemeine Weisungen im Bundesanzeiger veröffentlicht werden müssen. Was das für Einzelweisungen bedeutet, ist juristisch strittig, aber in der Praxis scheinen die Behörden hier de facto eine gewisse Unabhängigkeit und das entsprechende Selbstbewusstsein - zu haben.

Eine ähnliche Struktur wäre auch bei der Finanzaufsicht sinnvoll. Die Fokussierung auf Finanzstabilität und Anlegerschutz würde den Fokus auf das Wesentliche richten. Eine De-facto-Unabhängigkeit würde verhindern, dass die Finanzaufsicht als Instrument zur Förderung „nationaler Champions“ oder auch nur zum Schutz parafiskalischer Institutionen missbraucht wird.

3 Die BaFin ist allerdings unabhängig bei Tätigkeiten im Rahmen des Einheitlichen Europäischen Aufsichtsmechanismus, dies unter der Ägide der EZB.
Institutionelle Trennung von den beaufsichtigten Instituten durchsetzen

Auch die Nähe der BaFin zu den von ihr beaufsichtigten Instituten sollte beendet werden. Ein erster Schritt dazu wäre eine Reform des BaFin-Verwaltungsrats, in dem derzeit noch Branchenverbände und -mitglieder vertreten sind. Diese sollten keine Entscheidungsrechte über die BaFin haben und sollten nicht im BaFin-Verwaltungsrat vertreten sein.

\section{Anleger- und Verbraucherschutz in den Fokus nehmen}

Bisher hat Verbraucherschutz für die BaFin keine erkennbar wichtige Rolle gespielt. Insofern ist zu begrüßen, dass die Bundesregierung diesen Bereich stärken will. Es ist allerdings zu befürchten, dass im Konfliktfall der Verbraucherschutz doch wieder hintansteht.

In den USA wurde vor dem Hintergrund der Finanzkrise entschieden, den Verbraucherschutz im Finanzbereich in einer neuen, unabhängigen Behörde, dem Consumer Financial Protection Bureau (CFPB) zu bündeln, die mit einem Budget von rund 650 Mio. US-\$ schlagkräftig ausgestattet wurde. Diese Institution erhielt starke Aufsichts- und Durchgriffsrechte auf nahezu alle Finanzinstitute des Landes. Seit ihrem Start 2011 hat sie mehr als 12,4 Mrd. US-\$ an Schadenzahlungen von der Finanzindustrie erstritten, von denen 31 Mio. US-Bürger:innen profitieren konnten. Der Unterschied zur BaFin ist eklatant.

Eine eigene Behörde für Verbraucherschutz im Finanzsektor würde diesem Anliegen das erforderliche Gewicht geben. Will man nicht so weit gehen, so sollte der Verbraucherschutz wenigstens aus seiner derzeitigen Stellung in einer der Wertpapieraufsicht untergeordneten Abteilung herausgehoben und in eine eigene Exekutivdirektion gebracht werden. Diese wäre der Banken- oder der Wertpapieraufsicht gleichzustellen.

\section{Effektivität der Aufsichtstätigkeit}

\section{Effektivität der Abschreckung}

Die Wirksamkeit der Aufsicht hängt davon ab, dass sich die Finanzinstitute von sich aus an die Regeln halten, um Eingriffe und Sanktionen zu vermeiden. Die Passivität der BaFin gegenüber exorbitanten Risiken und Skandalen wirkt hochgradig kontraproduktiv. Folgende Punkte sind wichtig:

- Erhöhung des Entdeckungsrisikos durch besseren Whistleblowerschutz. Dazu gehören auch Zeugenschutzprogramme und finanzielle Entschädigungen wie in den USA. 
- Erhöhung der Qualität der Arbeit des zuständigen Personals durch entsprechende Rekrutierung und Motivation.

- Deutliche Verschärfung der Sanktionen für Fehlverhalten, z.B. nach dem Vorbild der Wettbewerbsbehörden.

\section{Zuständigkeitswirrwarr überwinden}

Es gibt zu viele verschiedene Behörden im Finanzsektor. Ein eklatantes Beispiel ist das behördliche Nebeneinander in der Geldwäscheprävention. Im Fall Wirecard glaubte die Bezirksregierung Niederbayern, die BaFin sei dafür zuständig, die BaFin dagegen, die Zuständigkeit liege bei der Bezirksregierung Niederbayern. Infolgedessen blieben beide untätig. Es ist auch nicht sinnvoll, dass die Überwachung der verschiedenen Börsengesellschaften bei Landesbehörden liegt, die Aufsicht über den Handel an diesen Börsen dagegen bei der BaFin.

In der Bankenaufsicht arbeiten Bundesbank und BaFin zusammen. Die Vor-Ort-Prüfungen der Banken werden von einer Abteilung der Bundesbank vorgenommen. Daher ist die Aufsichtsbehörde weit von den Aufsichtsobjekten entfernt und muss sich auf die Berichte der Bundesbank verlassen.

\section{Fazit}

Die Bürgerbewegung Finanzwende hat seit ihrer Gründung 2018 große Defizite der Aufsicht dokumentiert. Das Paradigma, wonach die Finanzaufsicht in gutem Einver- nehmen mit den Beaufsichtigten wirken soll, statt die Einhaltung der Regeln durch alle Beteiligten durchzusetzen, blieb nach 2008 erhalten und wurde auch durch spätere Skandale nicht erschüttert.

Es wäre zu wünschen, dass es wenigstens jetzt, nach dem Wirecard-Skandal, zu einem Paradigmenwechsel käme. Bisher allerdings wurde weder die Unabhängigkeit der BaFin noch der Verbraucherschutz maßgeblich gestärkt. Eine Abkehr von einer „Kuschelaufsicht“ hin zu einer „Aufsicht mit Biss“, wie sie Finanzminister Scholz forderte, würde mehr verlangen als die bisherigen Reformmaßnahmen: eine Neuausrichtung der Governance der BaFin mit größerer Distanz von Ministerium und Banken und stärkerem Verbraucherschutz sowie eine deutliche Stärkung der Effektivität durch wirksamere Abschreckungsmöglichkeiten und eine Bereinigung von Kompetenzwirrwarr.

\section{Literatur}

Asmussen, J. (2006), Verbriefungen aus der Sicht des Bundesfinanzministeriums, Kredit und Kapital 19, 10-12.

Blank, P., A.-K. Kaufhold, J. P. Krahnen und K. Langenbucher (2021), BaFin (in)depence - A reform proposal, SAFE White Paper, 82, März.

BMF (Bundesministerium der Finanzen) (2021), Mehr Biss für die Finanzaufsicht, https://www.bundesfinanzministerium.de/Content/DE/Standardartikel/Themen/Internationales_Finanzmarkt/Finanzmarktpolitik/202102-02-mehr-biss-fuer-die-finanzaufsicht.html (5. Juli 2021).

Hellwig, M. F. (2017), Finanzstabilität und Verantwortlichkeit - Stellungnahme für das Bundesverfassungsgericht, Kredit und Kapital, 50, 421-454.

Schick, G. (2020), Die Bank gewinnt immer, Campus-Verlag, 32.

Thiemann, M. (2012), Out of the Shadow? Accounting for Special Purpose Entities in European Banking Systems, Competition and Change, 16, 37-55. 